\title{
An SIR rumor pairwise model with spreading age
}

\author{
Yao Liu*, Jinxian Li \\ School of Mathematical Sciences, Shanxi University, Taiyuan, 030006, P. R. China.
}

\begin{abstract}
In this paper, we extend the classical rumor model with non-Markovian recovery process in a complex network. We follow the ideas from Röst to analyze the pairwise model; then, the hyperbolic system can be transformed into integro-differential equations. For the rumor model, the reproduction number is obtained. Next, we use numerical simulation to verify the accuracy of the result. In the end, we focus on how the three different distributions of recovery time with spreading age influence on rumor model. The result illustrates the significant effect of different distribution functions on the process of rumor spreading.
\end{abstract}

Keywords: Rumor pairwise model, spreading age, reproduction number, numerical simulation.

2020 MSC: 05C82, 37N25, 92D25.

(C)2021 All rights reserved.

\section{Introduction}

The rumor is defined as a kind of social phenomenon that the wrong information spreads on a large scale in a short time through chains of communication. Like the epidemic model, we can understand the mechanism of rumor transmission by establishing mathematical model, and find effective methods to reduce the harm that caused by the rumor. In 1965 years, Daley and Kendall [3] proposed the classical dynamic model of the rumor in which the population was divided into three groups. But the distribution and connection of the population are chaotic in the real society, they cannot be described by classical models. After that, Chen [1] modified the SIR model and proposed the ILSCR rumor spreading model. He found the spreaders might exist a cooling-off period before becoming the stiflers and the population would move in a certain area. Huo et al. [7] introduced a dynamic model for the rumor spreading called I2SR, they considered the activity of nodes and divided spreaders into the high rate of active state and the low rate of active spreaders. Since the rumor spread faster, we usually ignore demographic changes, such as birth and death. The hesitating mechanism, refutation and self-growth mechanism are introduced into the rumor model $[5,11,18]$. Considering the effect of medias, such as social software or network platform, some researchers [8] proposed a nonlinear rumor mathematical model to describe the impact of media report on rumor spreading. The feasible equilibriums and their stability properties were analyzed, then the numerical model was used to prove the effectiveness of their optimal control method. On that basis,

\footnotetext{
*Corresponding author

Email addresses: 2502653167@qq. com (Yao Liu), Ljxsmile1@163.com (Jinxian Li)
}

doi: 10.22436/jnsa.014.03.04

Received: 2020-07-05 Revised: 2020-08-07 Accepted: 2020-08-17 
Hosni et al. [4] analyzed the impact of online social network addiction on the propagation of the rumor, and they extended the classical SIR model to a new model with the addicted individuals. The classic SIR model was extended to many new models, such as ILSCR model [1], SE2IR model [18], I2S2R model [22], SEIR model [12] and SDILR model [19]. Assuming the spreading process of the rumor is non-Markovian behavior, Chunlong et al. [2] modified the SEIR model and gave the formula to calculate the spreading rate. Moreover, Huo et al. [6] regarded the process that the official denied the rumor as a periodic impulse and proposed a XWYZ rumor spreading model with time delay and impulse vaccination.

In many cases, we realize demography and infection age are important factors in the epidemic disease model $[9,21]$. However, in the literature [10], it found that the most of the researcher ignored the important effect of the spreading age on the spreading process of the rumor. In general, they assumed the spreading rate and recovery rate of the rumor or the disease was constant, which meant the spreading and recovery process was Markovian behavior. Obviously, these are not consistent with the reality [14, 17]. To make the rumor model more accurate and truthful, after investigating the modern epidemic dynamics models and documents $[9,14,21]$, we introduce the risk function instead of the recovery rate, where the recovery rate depend on the spreading age. Numerical simulations are used to verify the accuracy of the model and ensure the model in line with the reality. In numerical simulations, Jing et al. [9] and Zhang et al. [21] discovered that the different recovery time distributions could lead to different effects on the spreading process. Neil et al. [14] studied the rumor models on scale-free network by performing Monte Carlo simulations and made numerical solutions of the mean-field equations. Furthermore, Wilkinsion et al. [16] provided an upper bound for the expected epidemic scale at any fixed time $t>0$ and Jing et al. [9] found that increasing the variance in the recovery time or decreasing the variance in infection period could effectively control the diseases.

The present paper extend the Röst SIR model [13] and the other literature [9, 15, 21] to establish a rumor pairwise model and analyze the effect of spreading age on the speed of the rumor transmission in complex network. The rest of this article is arranged as following. The rumor model with spreading age is given in Section 2. In Section 3, we follow the ideas of Yang et al. [20] to calculate the reproduction number. In Section 4, we investigate the effect of different recovery time distributions (Gamma, Uniform and Weibull distributions) on the reproduction number and the final scale by numerical simulations. Moreover, we find the mean length of the spreading period will affect the peak time of the rumor outbreak, the duration of the rumor, the peak value of spreading individuals and the final scale. Our present findings are summarized in Section 5.

\section{The model formulation}

In this section, we consider a complex network with $\mathrm{N}$ nodes and average degrees $\mathrm{n}$. So we get an undirected graph $G=(V, E), V$ is the set of all nodes, $E$ is the set of all the edges. According to the definition of Daley and Kendall [3], they classify individuals into three classes: spreading individual who is transmitting the rumor, called spreader, ignorant individual who do not know the rumor and vulnerable to be affected, known as ignorant people, removed individual who knows the rumor but do not transmit it, called stifler. For convenience, we use S, I, and R to denote ignorant people, spreader, and stifler, respectively. The rules of rumor model are: firstly, when an ignorant people meets a spreader, ignorant people become spreader at the rate of $\lambda, \lambda$ is the spreading rate. Secondly, because of the different educational level of the ignorant people, when they meet the spreader, the ignorant people will convert to stifler at the rate of $\mu$. Thirdly, when the spreader meets the spreader or stifler, the initial spreader is converted to stifler at the rate of $\gamma$, which depends on spreading age.

The notations are similar to those notations given in the literature [21], the explanations of variables and parameters are shown in Table 1 . Then we can obtain

$$
[\mathrm{I}](\mathrm{t})=\int_{0}^{\infty} i(t, a) d a, \quad[S I](t)=\int_{0}^{\infty} \operatorname{Si}(t, a) d a, \quad[\operatorname{ISI}](t)=\int_{0}^{\infty} \operatorname{ISi}(t, a) d a, \quad[S I S](t)=\int_{0}^{\infty} \operatorname{Si}(t, a) S d a .
$$

Since the recovery process is non-Markovian process with cumulative distribution function $F(a)$ and 
probability density function $f(a)$, following the definition from literature $[13,14,21]$, let $\xi(a)=1-F(a)$ is the survival function. Then hazard function $\gamma(a)=-\frac{\dot{\xi}(a)}{\xi(a)}=\frac{f(a)}{\xi(a)}$ is the recovery rate. Furthermore, $\gamma(\mathrm{a})$ is bounded on the interval $\mathbb{R}_{+}=[0, \infty)$.

Table 1: Description of variables and parameters

\begin{tabular}{ll}
\hline Notations & Interpretations \\
\hline$[X],[X Y],[X Y Z]$ & $\begin{array}{l}\text { the expected number of nodes in state } X, \text { links in state } X-Y, \text { triplets in state } X-Y-Z \\
\text { at time } t, \text { respectively, } X, Y, Z \in\{S, I, R\}\end{array}$ \\
$\lambda$ & the spreader transmits the rumor to its neighbors with probability $\lambda$ \\
$\mu$ & the ignorant turn into the stifler with probability $\mu$ \\
$\gamma(a)$ & the recovery rate of spreading individual with spreading age a \\
$i(t, a)$ & the density of spreading nodes with respect to spreading age $a$ at time $t$ \\
$S i(t, a)$ & the density of $S-i$ links, where the spreading node $i$ has spreading age $a$ at time $t$ \\
$I S i(t, a)$ & the density of $I-S-i$ triplets, where the spreading node $i$ has spreading age $a$ at time $t$ \\
$\varphi(a)$ & the density function of spreading nodes with respect to spreading age a at the initial time \\
$N$ & the number of total population
\end{tabular}

The rumor model with Markovian spreading process and non-Markovian recovery process satisfies the equation

$$
\left\{\begin{array}{l}
{[\dot{S}](t)=-\lambda[S I](t)-\mu[\operatorname{SI}](t),} \\
{[\dot{S S}](t)=-2 \lambda[S S I](t)-2 \mu[S S I](t),} \\
\left(\frac{\partial}{\partial t}+\frac{\partial}{\partial a}\right) i(t, a)=-\gamma(a)(\operatorname{Ii}(t, a)+\operatorname{Ri}(t, a)), \\
\left(\frac{\partial}{\partial t}+\frac{\partial}{\partial a}\right) \operatorname{Si}(t, a)=-(\lambda+\mu) \operatorname{Si}(t, a)-\gamma(a)(\operatorname{Si}(t, a) I+\operatorname{Si}(t, a) R)-(\lambda+\mu) \operatorname{ISi}(t, a) .
\end{array}\right.
$$

Substituting the following relations given in [21] and the following closure approximations [13] into system (2.1),

$$
\begin{aligned}
n i(t, a) & =\operatorname{Si}(t, a)+\operatorname{Ii}(t, a)+\operatorname{Ri}(t, a), \\
n S i(t, a) & =S i(t, a) S+\operatorname{Si}(t, a) I+\operatorname{Si}(t, a) R, \\
n I i(t, a) & =\operatorname{Ii}(t, a) S+\operatorname{Ii}(t, a) I+\operatorname{Ii}(t, a) R,
\end{aligned}
$$

and

$$
[X Y Z](t)=\frac{[X Y](t)[Y Z](t)}{[Y](t)}, X, Y, Z \in\{S, I, R\},
$$

we can reduce system (2.1) to the following four-dimensional system

$$
\left\{\begin{array}{l}
{[\dot{S}](t)=-\lambda[S I](t)-\mu[S I](t)} \\
{[\dot{S S}](t)=-2 \lambda \frac{[S S](t)[S I](t)}{[S](t)}-2 \mu \frac{[S S](t)[S I](t)}{[S](t)}} \\
\left(\frac{\partial}{\partial t}+\frac{\partial}{\partial a}\right) i(t, a)=-\gamma(a)(n i(t, a)-S i(t, a))=-\gamma(a) n i(t, a)+\gamma(a) \operatorname{Si}(t, a) \\
\left(\frac{\partial}{\partial t}+\frac{\partial}{\partial a}\right) \operatorname{Si}(t, a)=-(\lambda+\mu+n \gamma(a)) \operatorname{Si}(t, a)-(\lambda+\mu) \frac{[S I](t) S i(t, a)}{[S](t)}+\gamma(a) \frac{S i(t, a)^{2}}{i(t, a)}
\end{array}\right.
$$

with the boundary conditions

$$
\left\{\begin{array}{l}
i(t, 0)=\lambda[S I](t) \\
\operatorname{Si}(t, 0)=\lambda[S S I](t)
\end{array}\right.
$$

The following will show the initial conditions for the equation (2.2). Let $\varphi(a)$ be the density function of spreading nodes with respect to spreading age $a$ at the initial time, and $\psi(a)$ is the density function 
of $[\mathrm{SI}]$ with respect to spreading age $a$ at the initial time $t$. According to biological meaning, we have $\lim _{a \rightarrow \infty} \varphi(a)=0$ and $\lim _{a \rightarrow \infty} \psi(a)=0$.

$$
\left\{\begin{array}{l}
{[S](0)=[S]_{0},} \\
{[S S](0)=[S S]_{0},} \\
i(0, a)=\varphi(a), \\
\operatorname{Si}(0, a)=\psi(a),
\end{array}\right.
$$

where $[S]_{0},[S S]_{0} \in \mathbb{R}_{+}=[0, \infty)$, and $\varphi(a), \psi(a) \in \mathrm{L}_{+}^{1}$, and the initial conditions are determined by the initial network. Here $\mathrm{L}_{+}^{1}:[0, \infty) \rightarrow \mathbb{R}_{+}$is the space of function that is nonnegative and Lebesgue integrable over the specified interval. All the parameters are positive. Moreover, we assume that model (2.2) satisfies the compatibility conditions

$$
\varphi(0)=\lambda \int_{0}^{\infty} \psi(\mathrm{a}) \mathrm{da}, \quad \psi(0)=\lambda \frac{[\mathrm{SS}]_{0}}{[S]_{0}} \int_{0}^{\infty} \psi(\mathrm{a}) \mathrm{da} .
$$

For simplicity of notation, define $\pi: \mathbb{R}_{+} \rightarrow \mathbb{R}_{+}$by

$$
\sigma(a)=e^{\int_{0}^{a} \lambda+\mu+n \gamma(s) d s}
$$

and $v: \mathbb{R}_{+} \rightarrow \mathbb{R}_{+}$by

$$
v(t)=e^{\int_{0}^{t}(\lambda+\mu) \frac{[S I](s)}{[S](s)} d s} .
$$

Using the method of characteristics, system (2.2) is converted into the following integro-differential equations,

$$
\begin{aligned}
& {[\dot{S}](t)=-\lambda[S I](t)-\mu[S I](t),} \\
& {[S \operatorname{SS}](t)=-2 \lambda \frac{[S S](t)[S I](t)}{[S](t)}-2 \mu \frac{[S S](t)[S I](t)}{[S](t)},} \\
& {[\dot{I}](t)=\lambda[\operatorname{SI}](t)-\int_{0}^{t} n \gamma(a)\left\{\lambda[S I](t-a) e^{-\int_{0}^{a} n \gamma(s) d s}+\int_{0}^{a} \gamma(s) \operatorname{Si}(t-a+s, s) e^{-\int_{s}^{a} n \gamma(w) d w} d s\right\} d a} \\
& -\int_{t}^{\infty} n \gamma(a)\left\{\varphi(a-t) e^{-\int_{a-t}^{a} n \gamma(s) d s}+\int_{0}^{t} \gamma(a-t+s) \operatorname{Si}(s, a-t+s) e^{-\int_{a-t+s}^{a} n \gamma(w) d w} d s\right\} d a \\
& +\int_{0}^{t} \gamma(a)\left\{\frac{[S](t-a) v(t) \sigma(a)}{\lambda[S S](t-a)[S I](t-a) v(t-a)}-\int_{0}^{a} \frac{\gamma(s) v(t) \sigma(a)}{i(t-a+s, s) v(t-a+s) \sigma(s)} d s\right\}^{-1} d a \\
& +\int_{t}^{\infty} \gamma(a)\left\{\frac{v(t) \sigma(a)}{\psi(a-t) \sigma(a-t)}-\int_{0}^{t} \frac{\gamma(a-t+s) v(t) \sigma(a)}{i(s, a-t+s) v(s) \sigma(a-t+s)} d s\right\}^{-1} d a, \\
& {[\dot{S I I}](t)=\lambda \frac{n}{N}[S](t)[S I](t)-(\lambda+\mu)[S I](t)-(\lambda+\mu) \frac{[S I]^{2}(t)}{[S](t)}} \\
& +\int_{0}^{t} \frac{\gamma(a)}{\lambda[S I](t-a) e^{-\int_{0}^{a} n \gamma(s) d s}+\int_{0}^{a} \gamma(s) \operatorname{Si}(t-a+s, s) e^{-\int_{s}^{a} n \gamma(w) d w} d s} \\
& \left\{\frac{[S](t-a) v(t) \sigma(a)}{\lambda[S S](t-a)[S I](t-a) v(t-a)}-\int_{0}^{a} \frac{\gamma(s) v(t) \sigma(a)}{i(t-a+s, s) v(t-a+s) \sigma(s)} d s\right\}^{-2} d a \\
& +\int_{t}^{\infty} \frac{\gamma(a)}{\varphi(a-t) e^{-\int_{a-t}^{a} n \gamma(s) d s}+\int_{0}^{t} \gamma(a-t+s) S i(s, a-t+s) e^{-\int_{a-t+s}^{a} n \gamma(w) d w} d s} \\
& \left\{\frac{v(t) \sigma(a)}{\psi(a-t) \sigma(a-t)}-\int_{0}^{t} \frac{\gamma(a-t+s) v(t) \sigma(a)}{i(s, a-t+s) v(s) \sigma(a-t+s)} d s\right\}^{-2} d a \\
& -\int_{0}^{t} n \gamma(a)\left\{\frac{[S](t-a) v(t) \sigma(a)}{\lambda[S S](t-a)[S I](t-a) v(t-a)}-\int_{0}^{a} \frac{\gamma(s) v(t) \sigma(a)}{i(t-a+s, s) v(t-a+s) \sigma(s)} d s\right\}^{-1} d a \\
& -\int_{t}^{\infty} n \gamma(a)\left\{\frac{v(t) \sigma(a)}{\psi(a-t) \sigma(a-t)}-\int_{0}^{t} \frac{\gamma(a-t+s) v(t) \sigma(a)}{i(s, a-t+s) v(s) \sigma(a-t+s)} d s\right\}^{-1} d a .
\end{aligned}
$$

Dividing the second equation of (2.3) by the first one and then separating the variables, we have 


$$
[S S](t)=\frac{n}{N}[S]^{2}(t) .
$$

So it is clear that $[S S](t)$ can be replaced by $[S](t)$ in equation (2.4).

\section{Reproduction number}

In this section, we follow the methods from Yang et al. [20] to calculate the reproduction number. According to literature [20], we have obtained renewal equation from the fourth equation of system (2.3). Linearizing the equation at disease free equilibrium $\mathrm{E}_{0}=(\mathrm{N}, \mathrm{nN}, 0,0)$, we have

$$
\frac{d[S I](t)}{d t}=-(\lambda+\mu)[S I](t)+n \lambda[S I](t)-n \lambda \int_{0}^{t} n \frac{\gamma(a)}{\sigma(a)}[S I](t-a) d a .
$$

Solving the equation (3.1) yields

$$
[S I](t)=q^{N p}(t)+\int_{0}^{t}\left\{n \lambda[S I](s)-n \lambda \int_{0}^{s} n \frac{\gamma(a)}{\sigma(a)}[S I](s-a) d a\right\} e^{-(\lambda+\mu)(t-s)} d s .
$$

Denote $\mathrm{q}^{\mathrm{Np}}(\mathrm{t})=[\mathrm{SI}]_{0} e^{-(\lambda+\mu) \mathrm{t}}$ and let

$$
\widehat{[S I]}(w)=\int_{0}^{\infty} e^{-w t}[S I](t) d t \quad \text { and } \quad q^{N p(w)}=\int_{0}^{\infty} e^{-w t} q^{N p}(t) d t
$$

be Laplace transformation for $[\mathrm{SI}]$ and $\mathrm{q}^{\mathrm{Np}}$. Taking Laplace transformation on both sides of (3.2), we have

$$
\begin{aligned}
& \int_{0}^{\infty} e^{-w \mathrm{t}}[\mathrm{SI}](\mathrm{t}) \mathrm{dt}=\int_{0}^{\infty} e^{-w \mathrm{t}} \mathrm{q}^{\mathrm{Np}}(\mathrm{t}) \mathrm{dt}+\mathrm{n} \lambda \int_{0}^{\infty} e^{-w \mathrm{t}} \mathrm{dt} \int_{0}^{\mathrm{t}}[\mathrm{SI}](\mathrm{s}) \mathrm{e}^{-(\lambda+\mu)(\mathrm{t}-\mathrm{s})} \mathrm{ds} \\
& -n \lambda \int_{0}^{\infty} e^{-w t} d t \int_{0}^{t} e^{-(\lambda+\mu)(t-s)} d s \int_{0}^{s} n \frac{\gamma(a)}{\sigma(a)}[S I](s-a) d a, \\
& \widehat{[\mathrm{SI}]}(w)=\mathrm{q}^{\widehat{N p}(w)}+n \lambda \int_{0}^{\infty}[\mathrm{SI}](\mathrm{s}) \mathrm{e}^{(\lambda+\mu) s} \mathrm{ds} \int_{s}^{\infty} e^{-(w+\lambda+\mu) t} d t \\
& -n \lambda \int_{0}^{\infty} n \frac{\gamma(a)}{\sigma(a)} d a \int_{a}^{\infty}[\operatorname{SI}](s-a) e^{(\lambda+\mu) s} d s \int_{s}^{\infty} e^{-(w+\lambda+\mu) t} d t \\
& =\mathrm{q}^{\widehat{N p}(w)}+\frac{n \lambda}{w+\lambda+\mu} \int_{0}^{\infty} e^{-w s}[\operatorname{SI}](s) d s \\
& -\frac{n \lambda}{w+\lambda+\mu} \int_{0}^{\infty} n \frac{\gamma(a)}{\sigma(a)} e^{-w a} d a \int_{a}^{\infty}[S I](s-a) e^{-w(s-a)} d s,
\end{aligned}
$$

so, we have

$$
\widehat{[S I]}(w)=q^{\widehat{N p}(w)}+n \lambda \frac{\widehat{[S I}(w)}{\lambda+\mu+w}\left(1-\int_{0}^{\infty} n \frac{\gamma(a)}{\sigma(a)} e^{-w a} d a\right) .
$$

Denote $\widehat{\mathrm{H}(w)}=\frac{n \lambda}{\lambda+\mu+w}\left(1-\int_{0}^{\infty} n \frac{\gamma(\mathrm{a})}{\sigma(\mathrm{a})} e^{-w a} \mathrm{da}\right)$. Therefore, the reproduction number is defined by

$$
\mathrm{R}_{0}=\widehat{[\mathrm{H}]}(0)=\frac{\mathrm{n} \lambda}{\lambda+\mu}(1-\mathrm{K}),
$$

where $K=\int_{0}^{\infty} n \frac{\gamma(a)}{\sigma(a)} d a$

\section{Simulations}

In this simulation, we consider a Markovian transmission process with spreading rate $\lambda$, the recovery rate is determined by the recovery time distribution $f_{R}(t)$. Especially the instantaneous recovery rate at spreading age $a$ is given by the hazard function of $f_{R}(a)$, i.e.,

$$
\gamma(a)=\frac{f_{R}(a)}{1-F_{R}(a)}
$$

where $F_{R}(a)=\int_{0}^{a} f_{R}(t) d t$ is the cumulative distribution function of the recovery time. 
We use sensitivity analysis to analyze the impact of spreading age on rumor spreading and advice some effective ways to control it. The relevant studies are proposed in literature [9]. To prove the effects of different recovery time distributions on the reproduction number and the final scale, we adopt some cases in which the recovery time obeys Gamma, Uniform or Weibull distributions. Let $X$ be the time since the moment at which the spreading individual has become spreader, which is a random variable.

Gamma distribution: In this case, $X$ obey the Gamma distribution, the usual symbol of Gamma distribution is $G\left(a_{1}, b_{1}\right)$, where $a_{1}$ called shape parameter and $b_{1}$ is scale parameter. The expected value is $E(X)=a_{1} b_{1}$ and variance is $\operatorname{Var}(X)=a_{1} b_{1}^{2}$. The probability density function is

$$
f(x)= \begin{cases}\frac{x^{a_{1}-1} e^{-x / b_{1}}}{b_{1}^{a_{1}} \Gamma\left(a_{1}\right)}, & x \geqslant 0, \\ 0, & x<0,\end{cases}
$$

where $\Gamma\left(a_{1}\right)$ is Gamma function, and the recovery rate is

$$
\gamma(a)=\frac{a^{a_{1}-1} e^{-a / b_{1}}}{b_{1}^{a_{1}}\left(\Gamma\left(a_{1}\right)-\int_{0}^{a / b_{1}} \chi^{a_{1}-1} e^{-x} d x\right)}, \quad \text { for } a \geqslant 0 .
$$

When $a_{1}=1$, the Gamma distribution reduces to an exponential distribution, conforming to a Markovian recovery course. In this paper, let $E(X)=1$, then the Gamma distribution is defined by one parameter $a_{1}$. Weibull distribution: In this case, $X$ obey the Weibull distribution, denoted as $W\left(a_{2}, b_{2}\right)$. The expected value is $E(X)=a_{2} \Gamma\left(1+\frac{1}{b_{2}}\right)$ and the variance is $\operatorname{Var}(X)=a_{2}^{2}\left[\Gamma\left(1+\frac{2}{b_{2}}\right)-\left(\Gamma\left(1+\frac{1}{k}\right)\right)^{2}\right]$. In this paper, let $E(X)=1$, then the Weibull distribution is defined by one parameter $a_{2}$.

$$
f(x)= \begin{cases}\frac{\mathrm{a}_{2}}{\mathrm{~b}_{2}}\left(\frac{\mathrm{x}}{\mathrm{b}_{2}}\right)^{\mathrm{a}_{2}-1} e^{-\left(x / \mathrm{b}_{2}\right)^{\mathrm{a}_{2}},}, & x \geqslant 0, \\ 0, & x<0,\end{cases}
$$

and the recovery rate is

$$
\gamma(a)=\frac{a_{2}}{b_{2}}\left(\frac{a}{b_{2}}\right)^{a_{2}-1}, \text { for } \quad a \geqslant 0
$$

In this paper, let $E(X)=1$, then the Weibull distribution is defined by one parameter $a_{2}$.

Uniform distribution: In this case, $X$ obey the Uniform distribution, denoted as $U\left(a_{3}, b_{3}\right)$. The expected value is $E(X)=\frac{a_{3}+b_{3}}{2}$ and the variance is $\operatorname{Var}(X)=\frac{\left(b_{3}-a_{3}\right)^{2}}{12}$. The probability density function is

$$
f(x)= \begin{cases}\frac{1}{b_{3}-a_{3}}, & a_{3} \leqslant x \leqslant b_{3}, \\ 0, & \text { otherwise, }\end{cases}
$$

where $b_{3}>a_{3} \geqslant 0$, are shape parameters and scale parameters respectively, and the recovery rate is

$$
\gamma(a)=\frac{1}{b_{3}-a}, \quad \text { for } \quad a \in\left[a_{3}, b_{3}\right] .
$$

If no otherwise specified, let $E(X)=1$, then the Uniform distribution is defined by one parameter $a_{3}$.

In order to compare these distributions, we fix the average recovery time to $1 / \gamma_{0}$. Then

$$
b_{1}=\frac{1}{a_{1} \gamma_{0}}, \quad b_{2}=\left[\Gamma\left(1+\frac{1}{a_{2}}\right) \gamma_{0}\right]^{-1}, \quad b_{3}=\frac{2}{\gamma_{0}}-a_{3} .
$$

If no otherwise specified, the numerical simulations of model (2.1) are performed with $\lambda=0.8, \mu=0.2$, $\mathrm{N}=1000, \mathrm{n}=5$, and the initial values are $[\mathrm{S}]_{0}=990,[\mathrm{I}]_{0}=10$.

To describe the final scale of the rumor spreading, we adopt the cumulative incidence of transmission, defined as the number of spreading or recovered nodes [15], which is denoted as J. Then we have

$$
\mathrm{J}=\mathrm{N}-[\mathrm{S}]_{\infty} .
$$

\subsection{The effect of the different recovery time distribution on rumor spreading}

In this section, we study the effects of the recovery time distribution on the rumor spreading in more detail. Firstly, we study the effect of the parameters of the recovery time distribution. 
For three types of recovery time distributions with $E(X)=1$, Fig. 1 shows the time series of the number of spreading individual with different parameters $a_{j}$. The graph displays the number of spreading individual presents an increasing tendency firstly, and then a downward trend for any parameters $a_{j}$. In addition, the number of spreading individual will reach its maximum earlier which becomes larger with the increase in $a_{j}$.

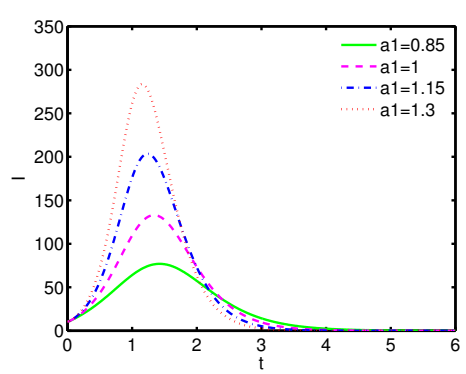

(a)

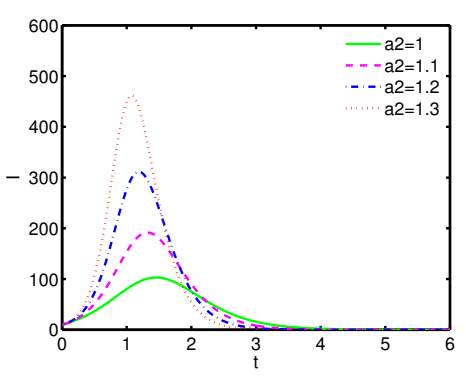

(b)

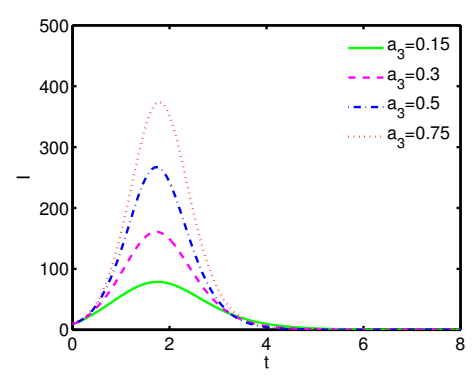

(c)

Figure 1: The time series of the number of the spreading individual for the different recovery time distributions with different parameters $a_{j}$ : (a) Gamma distribution; (b) Weibull distribution; (c) Uniform distribution.

Furthermore, we show the three-dimensional diagrams of the number of the spreading individuals with respect to time $t$ and spreading age a obeys Gamma, Weibull and Uniform distribution recovery time in Figs. 2-4. Suppose $E(X)=1$, then Figs. 2-4 show that they have the similar trend with given spreading age $a$ or time $t$, the number of spreader is going up to the peak and then going down over time when we fix spreading age $a$, while it is dropping with spreading age $a$ when we fix time $t$.

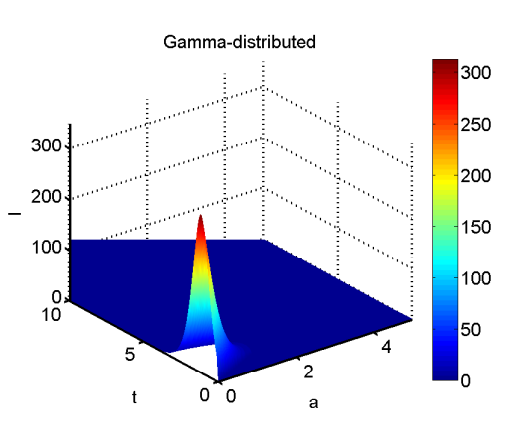

(a)

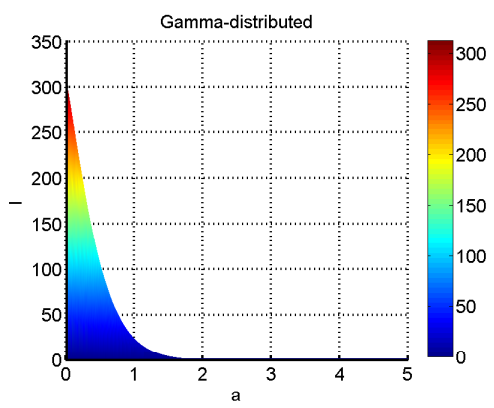

(c)

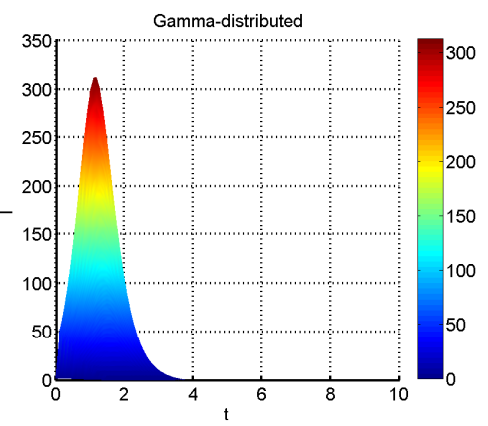

(b)

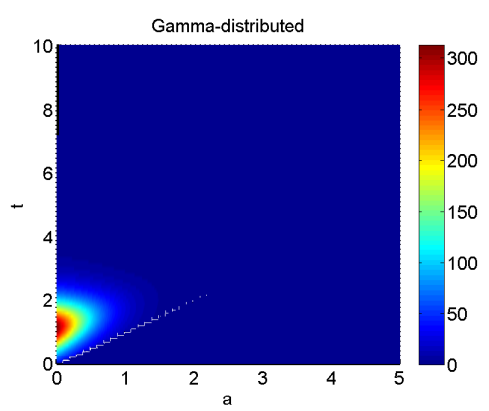

(d)

Figure 2: (a) Three-dimensional diagrams of spreading individuals with time $t$ and spreading age a for Gamma distribution, where $a_{1}=b_{1}=1$. (b) The time series of the number of the spreading individuals. (c) The spreading age series of the number of the spreading individuals. (d) The planform of the number of the spreading individual with time $t$ and spreading age a for Gamma distribution, where $a_{1}=1$. 


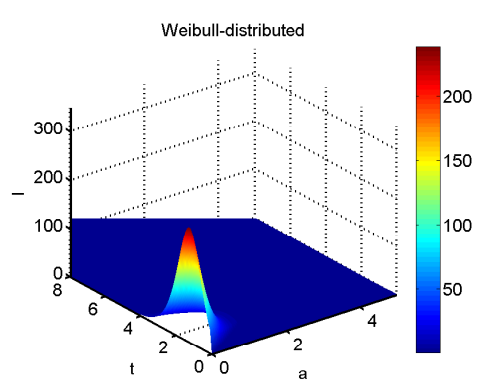

(a)

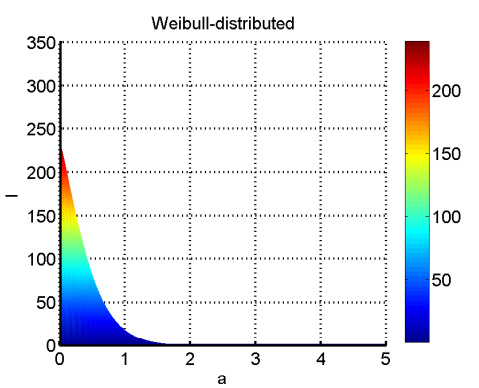

(c)

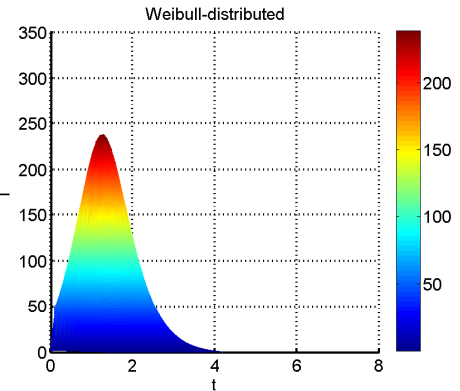

(b)

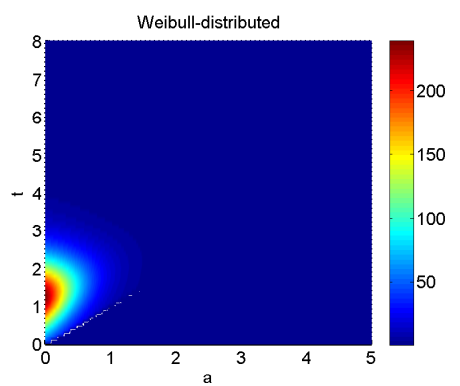

(d)

Figure 3: (a) Three-dimensional diagrams of spreading individuals with time $t$ and spreading age a for Weibull distribution, where $a_{2}=b_{2}=1$. (b) The time series of the number of the spreading individuals. (c) The spreading age series of the number of the spreading individuals. (d) The planform of the number of spreading individual with time $t$ and spreading age a for Weibull distribution, where $a_{2}=1$.

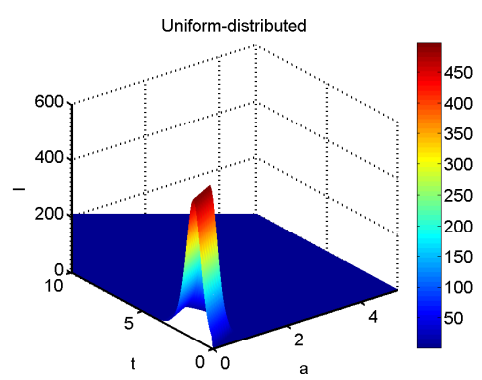

(a)

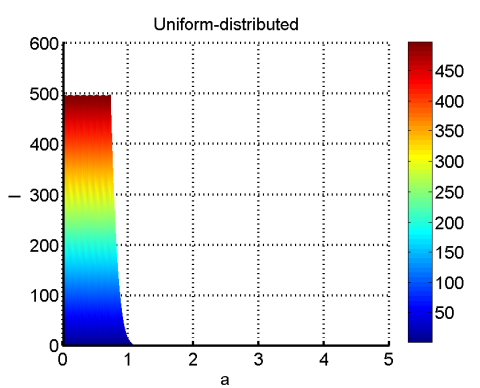

(c)

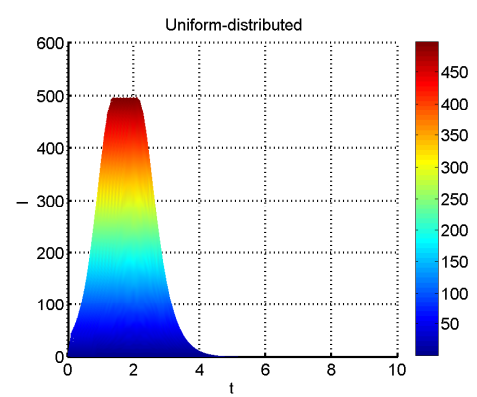

(b)

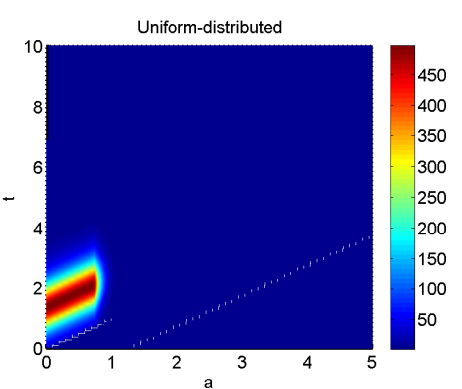

(d)

Figure 4: (a) Three-dimensional diagrams of spreading individuals with time $t$ and spreading age a for Uniform distribution, where $a_{3}=0.75, b_{3}=1.25$. (b) The time series of the number of the spreading individuals. (c) The spreading age series of the number of the spreading individuals. (d) The planform of the number of spreading individual with time $t$ and spreading age a for Uniform distribution, where $a_{3}=0.75$. 
What's more, we also analyze some other simulations on the final scale when the recovery time distribution obeys Gamma, Weibull and Uniform distribution. Let's $E(X)=1$, the total population $N=$ 1000 and the average degree $n=5$, the parameters $a_{j}=1, j=1,2,3$. When the spreading rate takes $\lambda=0.05$, the rumor can't spread widely among the crowd, the time series of ignorant people and spreader are given when the initial values are different (see Figs. 5-6). For the Fig. 5, it shows that a few of the population will be affected by the rumor no matter which distribution the recovery time obeys Gamma, Weibull or Uniform. In addition, when recovery time distribution obeys Uniform, the number of the affected population will be more than that when the recovery time distribution obeys Gamma, Weibull, because the number of spreader is going down more slowly (details in Fig. 6 (a)-(c)). Moreover, when the spreading rate takes $\lambda=0.8$, the rumors will erupt and spread among the crowd, the time series of the numbers of ignorant people and spreader are given, respectively, when the initial values are different (see Figs. 7-8). We can find that when the recovery distribution obeys Uniform, both the number of people affected by the rumor and the number of spreader are more than those when it obeys the other two distributions. Meanwhile, the mean length of the spreading period has influence on the final size, the peak and the duration of the rumor in different distributions. In Figs. 9-11 we find that the longer spreading period is, the longer the duration of an rumor is, the smaller the final rumor scale is, and the larger the peak value is.

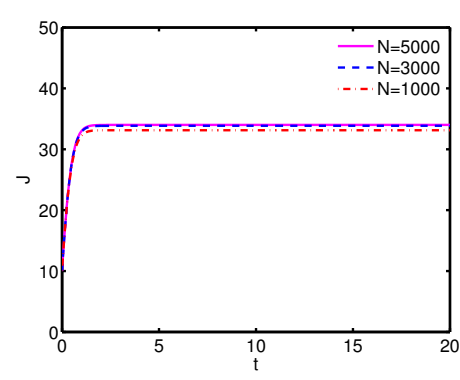

(a)

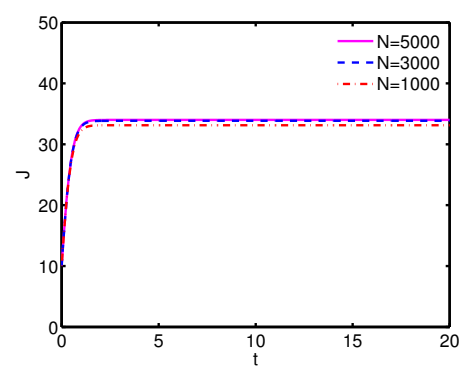

(b)

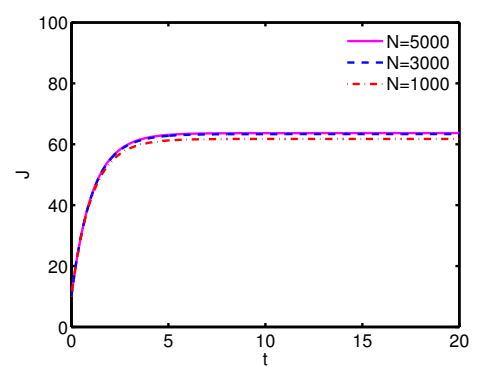

(c)

Figure 5: The time series of the the final scale for the different recovery time distribution with $\lambda=0.05, \mu=0.95, E=1$, $\mathrm{a}_{\mathrm{j}}=1, \mathrm{j}=1,2,3$ and the initial value $[\mathrm{I}]_{0}=10$. (a) Gamma distribution. (b) Weibull distribution. (c) Uniform distribution.

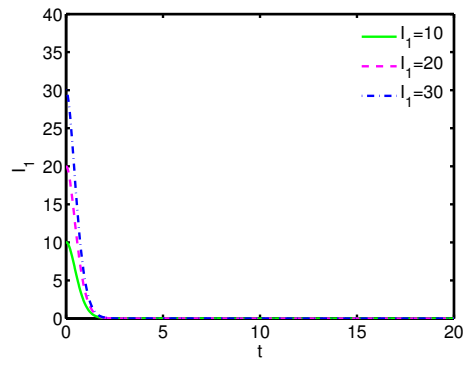

(a)

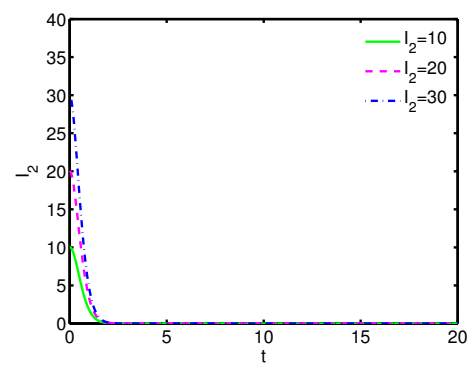

(b)

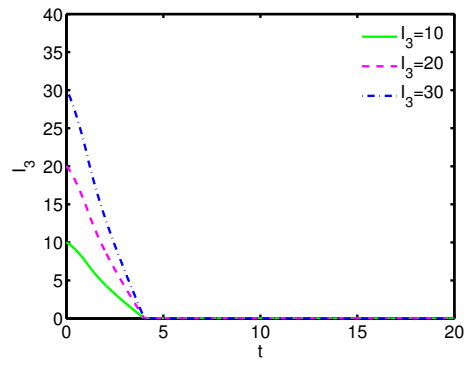

(c)

Figure 6: The time series of the number of the spreading individual for the different recovery time distributions with $\lambda=0.05$, $\mu=0.95, E=1, a_{j}=1, j=1,2,3$ and different initial values $I_{j}$. (a) Gamma distribution. (b) Weibull distribution. (c) Uniform distribution. 


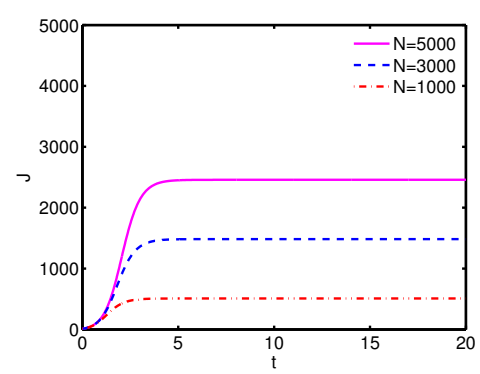

(a)

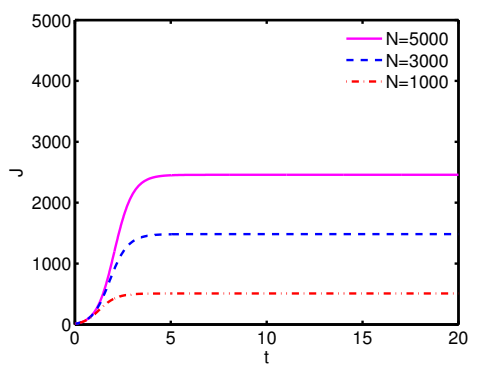

(b)

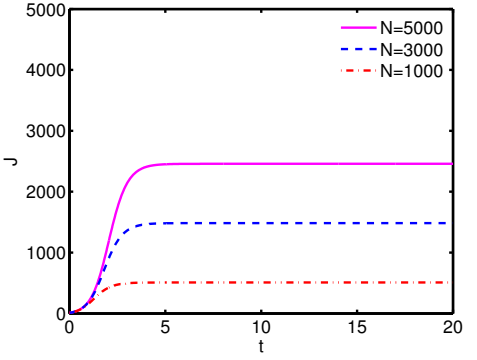

(c)

Figure 7: The time series of the the final scale for the different recovery time distribution with $\lambda=0.8, \mu=0.2, E=1$, $\mathrm{a}_{\mathrm{j}}=1, \mathrm{j}=1,2,3$ and the initial value $[\mathrm{I}]_{0}=10$. (a) Gamma distribution. (b) Weibull distribution. (c) Uniform distribution.

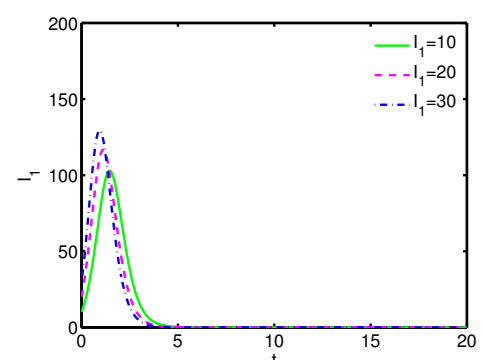

(a)

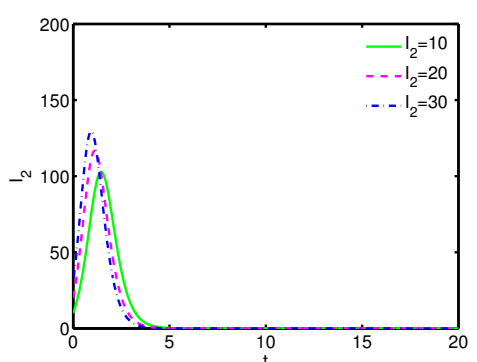

(b)

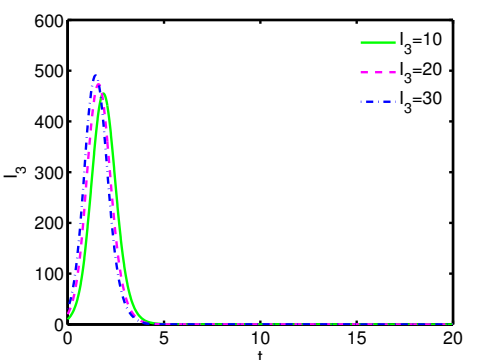

(c)

Figure 8: The time series of the number of the spreading individual for the different recovery time distributions with $\lambda=0.8$, $\mu=0.2, E=1, a_{j}=1, j=1,2,3$ and different initial values $I_{j}$. (a) Gamma distribution. (b) Weibull distribution. (c) Uniform distribution.

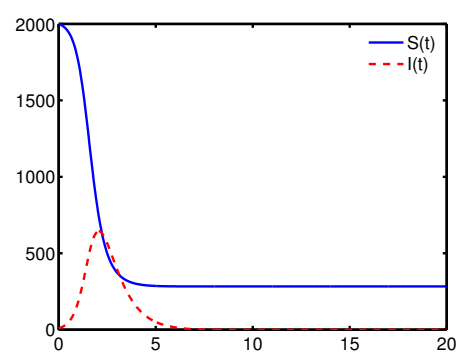

(a)

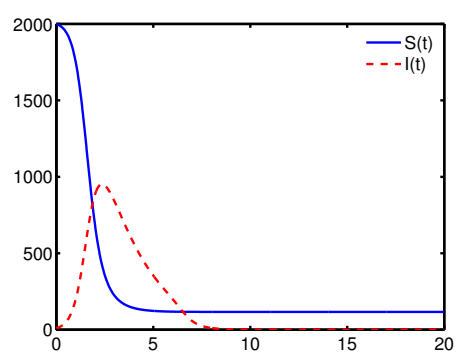

(b)

Figure 9: The time series of the number of the spreading individual and ignorant individual for Gamma distributions with $\lambda=0.8, a_{1}=b_{1}=1$ and the initial value $[S]_{0}=2000,[I]_{0}=10$. (a) The mean length of spreading period is four days. (b) The mean length of spreading period is ten days.

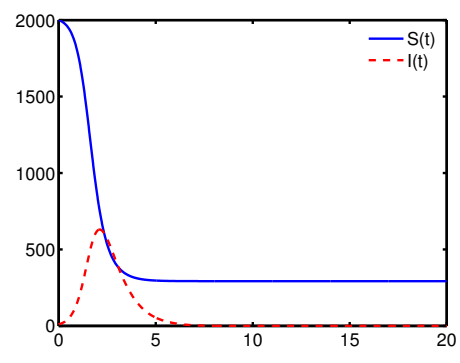

(a)

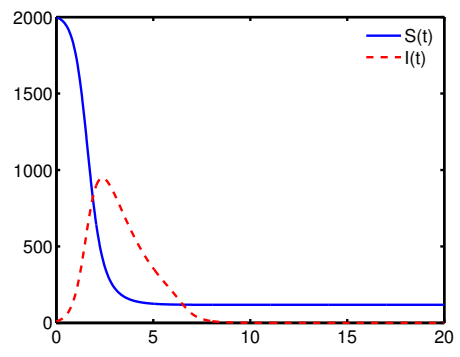

(b)

Figure 10: The time series of the number of the spreading individual and ignorant individual for Weibull distributions with $\lambda=0.8, a_{2}=b_{2}=1$ and the initial value $[S]_{0}=2000,[I]_{0}=10$. (a) The mean length of spreading period is four days. (b) The mean length of spreading period is ten days. 


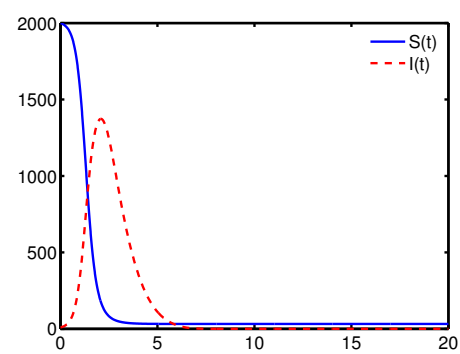

(a)

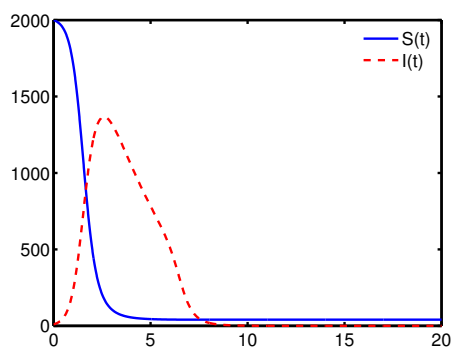

(b)

Figure 11: The time series of the number of the spreading individual and ignorant individual for Uniform distributions with $\lambda=0.8, a_{3}=0.75, b_{3}=1.25$ and the initial value $[S]_{0}=2000,[I]_{0}=10$. (a) The mean length of spreading period is four days. (b) The mean length of spreading period is ten days.

\section{5. conclusions}

In this paper, we mainly study and analyze the rumor propagation with the effect of spreading age. We obtain the dynamical model and the reproduction number. After that we analyze three different recovery time distributions on the spreading of the rumor. When the recovery time obeys Uniform distribution, the rumor spreading is the widest, under the same conditions $a_{i}=b_{i}=1, i=1,2,3, \lambda=0.8, \mu=0.2$, while Gamma distribution is smallest. Meanwhile, different shape parameters also have significant effects on the number of spreader. In addition, if $\lambda=0.05$, which means the reproduction number is less than one, the rumor shrinks directly and disappears eventually; if $\lambda=0.8$, which means the reproduction number is more than one, the rumor will spread for a while and then disappears. When the recovery time distribution and parameters are the same, the higher the propagation rate is, the more ignorant people will be affected. In the same distribution, compared with the shorter spreading periods, the longer mean of the spreading periods make the higher peak value and the larger the final scale. Therefore, the different means of the spreading periods can affect the peak value of spreader, the peak time of the rumor outbreak, the duration of the rumor and the final scale. So shortening the spreading periods provide us an effective way to control the spreading of the rumor. Therefore, on the one hand, the government and relevant departments should actively refute the rumor, on the other hand, they should improve the cultural level and discrimination ability of the masses.

The application of non-Markovian process in rumor model can provide a clearer direction for taking prediction measures. The method used in this paper following the ideas from Röst [13], can transform partial differential equations into integro-differential equations. It will help us to study more rumor and disease models with non-Markovian recovery process in complex network.

\section{Acknowledgment}

This work is supported by the National Natural Science Foundations of China under Grant. (Nos. 11571210, 11971279, 11331009, 11701348).

\section{References}

[1] G. Chen, ILSCR rumor spreading model to discuss the control of rumor spreading in emergency, Phys. A, 522 (2019), 88-97. 1

[2] F. Chunlong, S. Huimin, D. Guohui, Research on an Improved SEIR Network Rumor Propagation Model, J. Intell., 3 (2017), 86-91. 1

[3] D. J. Daley, D. G. Kendall, Stochastic Rumors, J. Inst. Math. Appl., 1 (1965), 42-55. 1, 2

[4] A. I. E. Hosni, K. Li, S. Ahmad, Analysis of the impact of online social networks addiction on the propagation of rumors, Phys. A, 542 (2020), 11 pages. 1 
[5] L. Huo, Y. Cheng, Dynamical analysis of a IWSR rumor spreading model with considering the self-growth mechanism and indiscernible degree, Phys. A, 536 (2019), 11 pages. 1

[6] L. Huo, C. Ma, Dynamical analysis of rumor spreading model with impulse vaccination and time delay, Phys. A, 471 (2017), 653-665. 1

[7] L. Huo, L. Wang, N. Song, Ch. Ma, B. He, Rumor spreading model considering the activity of spreaders in the homogeneous network, Phys. A, 468 (2017), 855-865. 1

[8] L. Huo, L. Wang, X. Zhao, Stability analysis and optimal control of a rumor spreading model with media report, Phys. A, 517 (2019), 551-562. 1

[9] W. Jing, Z. Jin, J. Zhang, An SIR pairwise epidemic model with infection age and demography, J. Biol. Dyn., 12 (2018), 486-508. 1, 4

[10] K. Kawachi, Deterministic models for rumor transmission, Nonlinear Anal. Real World Appl., 9 (2008), 1989-2028. 1

[11] R. Li, Y. Li, Z. Meng, Y. Song, G. Jiang, Rumor Spreading Model Considering Individual Activity and Refutation Mechanism Simultaneously, IEEE Access, 8 (2020), 63065-63067. 1

[12] Q. Liu, T. Li, M. Sun, The analysis of an SEIR rumor propagation model on heterogeneous network, Phys. A, 469 (2017), 372-380. 1

[13] G. Röst, Z. Vizi, I. Z. Kiss, Pairwise approximation for SIR type network epidemics with non-Markovian recovery, Proc. A., 474 (2018), 21 pages. 1, 2, 2, 5

[14] N. Sherborne, J. C. Miller, K. B. Blyuss, I. Z. Kiss, Mean-field models for non-Markovian epidemics on networks, J. Math. Biol., 76 (2018), 755-778. 1, 2

[15] E. Volz, SIR dynamics in random networks with heterogeneous connectivity, J. Math. Biol., 56 (2008), 293-310. 1, 4

[16] R. R. Wilkinson, F. G. Ball, K. J. Sharkey, The relationships between message passing, pairwise, Kermack-McKendrick and stochastic SIR epidemic models, J. Math. Biol., 75 (2017), 1563-1590. 1

[17] J. Yang, Y. Chen, Effect of infection age on an SIR epidemic model with demography on complex networks, Phys. A, 479 (2017), 527-541. 1

[18] H. Yao, X. Gao, SE2IR Invest Market Rumor Spreading Model Considering Hesitating Mechansim, J. Syst. Sci. Inform., 7 (2019), 54-69. 1

[19] Y. Yao, X. Xiao, C. Zhang, C. Dou, S. Xia, Stability analysis of an SDILR model based on rumor recurrence on social media, Phys. A, 535 (2019), 13 pages. 1

[20] J. Yang, F. Xu, The Computational Approach for the Basic Reproduction Number of Epidemic Models on Complex Networks, IEEE Acess, 7 (2019), 26474-26479. 1, 3

[21] J. Zhang, D. Li, W. Jing, Z. Jin, H. Zhu, Transmission dynamics of a two-strain pairwise model with infection age, Appl. Math. Model., 71 (2019), 656-672. 1, 2, 2

[22] Y. Zhang, J. Zhu, Dynamic behavior of an I2S2R rumor propagation model on weighted contract networks, Phys. A, 536 (2019), 20 pages. 1

\section{Appendix A: derivation of equations of second-order motifs for SIR model}

In this appendix, we give the derivation of $i(t, a)$ and $\operatorname{Si}(t, a)$. Firstly, we consider the following first-order PDE:

$$
\left(\frac{\partial}{\partial t}+\frac{\partial}{\partial a}\right) i(t, a)=-\gamma(a) n i(t, a)+\gamma(a) \operatorname{Si}(t, a),
$$

with boundary condition $i(t, 0)=\lambda[S I](t)$ and initial condition $i(0, a)=\varphi(a)$. In the method of characteristics, it is assumed that the partial differential equation can be express as a system of ordinary differential equations along the characteristic curves. The characteristic curves are found by solving the following ordinary differential equations:

$$
\frac{d t}{d \tau}=1, \quad \frac{d a}{d \tau}=1, \quad \frac{d y(\tau)}{d \tau}=-\gamma(a(\tau)) n y(\tau)+\gamma(a(\tau)) \operatorname{Si}(t(\tau), a(\tau))
$$

with initial conditions

$$
t(0)=t_{0}, \quad a(0)=a_{0}, \quad y(0)=i\left(t_{0}, a_{0}\right),
$$

where $y(\tau)=\mathfrak{i}(t(\tau), a(\tau))$. That the solution of system of ordinary differential equation along the characteristic curves is

$$
\begin{aligned}
& t(\tau)=t_{0}+\tau, \quad a(\tau)=a_{0}+\tau \\
& y(\tau)=y(0) e^{-\int_{0}^{\tau} n \gamma(a(s)) d s}+\int_{0}^{\tau} \gamma(a(s)) \operatorname{Si}(t(s), a(s)) e^{-\int_{s}^{\tau} n \gamma(a(w)) d w} d s
\end{aligned}
$$


Furthermore, it has

$$
i(t, a)=i\left(t_{0}, a_{0}\right) e^{-\int_{0}^{\tau} n \gamma\left(a_{0}+s\right) d s}+\int_{0}^{\tau} \gamma\left(a_{0}+s\right) \operatorname{Si}\left(t_{0}+s, a_{0}+s\right) e^{-\int_{s}^{\tau} n \gamma\left(a_{0}+w\right) d w} d s .
$$

If $t>a$, set $a_{0}=0$. Then $\tau=a$ and $t_{0}=t-a$. Substituting these expressions into equation (A.1), we obtain the solution when $t>a$

$$
i(t, a)=i(t-a, 0) e^{-\int_{0}^{a} n \gamma(s) d s}+\int_{0}^{a} \gamma(s) \operatorname{Si}(t-a+s, s) e^{-\int_{s}^{a} n \gamma(w) d w} d s .
$$

If $t \leqslant a$, set $t_{0}=0$. Then $\tau=t$ and $a_{0}=a-t$. Substituting these expressions into equation (A.1), we obtain the solution when $t \leqslant a$

$$
i(t, a)=i(0, a-t) e^{-\int_{a-t}^{a} n \gamma(s) d s}+\int_{0}^{t} \gamma(a-t+s) \operatorname{Si}(s, a-t+s) e^{-\int_{a-t+s}^{a} n \gamma(w) d w} d s .
$$

So finally, we substitute initial and boundary values into the expression (A.2) and (A.3), the solution of equation can be written in the form

$$
i(t, a)= \begin{cases}\lambda[\operatorname{SI}](t-a) e^{-\int_{0}^{a} n \gamma(s) d s}+\int_{0}^{a} \gamma(s) \operatorname{Si}(t-a+s, s) e^{-\int_{s}^{a} n \gamma(w) d w} d s, & t>a, \\ \varphi(a-t) e^{-\int_{a-t}^{a} n \gamma(s) d s}+\int_{0}^{t} \gamma(a-t+s) \operatorname{Si}(s, a-t+s) e^{-\int_{a-t+s}^{a} n \gamma(w) d w} d s, & t \leqslant a .\end{cases}
$$

Similarly, we give the derivation of $\operatorname{Si}(t, a)$.

$$
\left(\frac{\partial}{\partial t}+\frac{\partial}{\partial a}\right) \operatorname{Si}(t, a)=-(\lambda+\mu+n r(a)) \operatorname{Si}(t, a)-(\lambda+\mu) \frac{[\operatorname{SI}](t) \operatorname{Si}(t, a)}{[S](t)}+\gamma(a) \frac{\operatorname{Si}(t, a)^{2}}{i(t, a)},
$$

replacing $f(t)$ with $(\lambda+\mu) \frac{[S I](t)}{[S](t)}, g(a)$ with $(\lambda+\mu+n r(a))$ and $h(t, a)$ with $\frac{\gamma(a)}{i(t, a)}$, in our case we have

$$
\frac{d t}{d \tau}=1, \quad \frac{d a}{d \tau}=1, \quad \frac{d y(\tau)}{d \tau}=-f(t(\tau)) y(\tau)-g(a(\tau)) y(\tau)+h(t(\tau), a(\tau)) y^{2}(\tau),
$$

where $y(\tau)=\operatorname{Si}(t(\tau), a(\tau))$, the above equation is Bernoulli differential equation, so we divide both sides by $y^{2}(\tau)$, then let $y^{-1}(\tau)$ equal to $z(\tau)$, we get

$$
\frac{d z(\tau)}{d \tau}=f(t(\tau)) z(\tau)+g(a(\tau)) z(\tau)-h(t(\tau), a(\tau))
$$

The solution of the system of ordinary differential equations along the characteristic curves is

$$
z(\tau)=z(0) e^{\int_{0}^{\tau}[f(t(s))+g(a(s))] d s}-\int_{0}^{\tau} h(t(s), a(s)) e^{\int_{s}^{\tau}[f(t(w))+g(a(w))] d w} d s .
$$

Furthermore, it has

$$
\frac{1}{\operatorname{Si}(t, a)}=\frac{1}{\operatorname{Si}\left(t_{0}, a_{0}\right)} e^{\int_{0}^{\tau}[f(t(s))+g(a(s))] d s}-\int_{0}^{\tau} h(t(s), a(s)) e^{\int_{s}^{\tau}[f(t(w))+g(a(w))] d w} d s .
$$

Hence, simplifying the expression (A.4), we have

$$
S i(t, a)=\left[S i^{-1}\left(t_{0}, a_{0}\right) e^{\int_{0}^{\tau}\left[f\left(t_{0}+s\right)+g\left(a_{0}+s\right)\right] d s}-\int_{0}^{\tau} h\left(t_{0}+s, a_{0}+s\right) e^{\int_{s}^{\tau}\left[f\left(t_{0}+w\right)+g\left(a_{0}+w\right)\right] d w} d s\right]^{-1} .
$$

If $t>a$, set $a_{0}=0$, then $\tau=a$ and $t_{0}=t-a$, substituting boundary into equation, we have

$$
\operatorname{Si}(t, a)=\left\{\frac{[S](t-a) v(t) \sigma(a)}{\lambda[S S](t-a)[S I](t-a) v(t-a)}-\int_{0}^{a} \frac{\gamma(s) \sigma(a) v(t)}{i(t-a+s, s) \sigma(s) v(t-a+s)} d s\right\}^{-1} .
$$


If $t \leqslant a$, set $t_{0}=0$, then $\tau=t$ and $a_{0}=a-t$, substituting initial into equation, we have

$$
\operatorname{Si}(t, a)=\left\{\frac{v(t) \sigma(a)}{\psi(a-t) \sigma(a-t)}-\int_{0}^{t} \frac{\gamma(a-t+s) v(t) \sigma(a)}{i(s, a-t+s) v(s) \sigma(a-t+s)} d s\right\}^{-1} .
$$

The solution of equation can be written in the form

$$
\operatorname{Si}(t, a)= \begin{cases}\left\{\frac{[S](t-a) v(t) \sigma(a)}{\lambda[S S](t-a)[S I](t-a) v(t-a)}-\int_{0}^{a} \frac{\gamma(s) \sigma(a) v(t)}{i(t-a+s, s) \sigma(s) v(t-a+s)} d s\right\}^{-1}, & t>a \\ \left\{\frac{v(t) \sigma(a)}{\psi(a-t) \sigma(a-t)}-\int_{0}^{t} \frac{\gamma(a-t+s) v(t) \sigma(a)}{i(s, a-t+s) v(s) \sigma(a-t+s)} d s\right\}^{-1}, & t \leqslant a .\end{cases}
$$

\section{Appendix B: calculations for model derivation}

In this appendix, we give the derivation of $[\dot{I}](t)$ and $[\dot{S} I](t)$. Using $[I](t)=\int_{0}^{\infty} i(t, a) d a$, from the third equation of system (2.2) we have

$$
\begin{aligned}
{[\dot{\mathrm{I}}](\mathrm{t})=\int_{0}^{\infty} \frac{\partial}{\partial t} \mathfrak{i}(t, a) d a } & =\int_{0}^{\infty}\left(-\gamma(a) n i(t, a)+\gamma(a) \operatorname{Si}(t, a)-\frac{\partial}{\partial a} i(t, a)\right) d a \\
& =-\int_{0}^{\infty} n \gamma(a) i(t, a) d a+\int_{0}^{\infty} \gamma(a) \operatorname{Si}(t, a) d a-i(t, \infty)+i(t, 0) .
\end{aligned}
$$

Solving the first-order linear partial differential equation of $i(t, a)$ along characteristic lines, where the $i(t, a)$ is derived in detail in Appendix $A$, we have

$$
i(t, a)= \begin{cases}\lambda[\operatorname{SI}](t-a) e^{-\int_{0}^{a} n \gamma(s) d s}+\int_{0}^{a} \gamma(s) \operatorname{Si}(t-a+s, s) e^{-\int_{s}^{a} n \gamma(w) d w} d s, & t>a \\ \varphi(a-t) e^{-\int_{a-t}^{a} n \gamma(s) d s}+\int_{0}^{t} \gamma(a-t+s) \operatorname{Si}(s, a-t+s) e^{-\int_{a-t+s}^{a} n \gamma(w) d w} d s, & t \leqslant a .\end{cases}
$$

Applying the formula (A.6) for $[I](t)=\int_{0}^{\infty} i(t, a) d a$, we have

$$
\begin{aligned}
{[I](t)=} & \int_{0}^{t}\left\{\lambda[S I](t-a) e^{-\int_{0}^{a} n \gamma(s) d s}+\int_{0}^{a} \gamma(s) \operatorname{Si}(t-a+s, s) e^{-\int_{s}^{a} n \gamma(w) d w} d s\right\} d a \\
& +\int_{t}^{\infty}\left\{\varphi(a-t) e^{-\int_{a-t}^{a} n \gamma(s) d s}+\int_{0}^{t} \gamma(a-t+s) \operatorname{Si}(s, a-t+s) e^{-\int_{a-t+s}^{a} n \gamma(w) d w} d s\right\} d a .
\end{aligned}
$$

Finally, using that along the characteristic lines, $i(t, \infty)=\mathfrak{i}(0, \infty)=\varphi(\infty)=0$ from the assumption, substituting (A.6) and the boundary condition of $i(t, a)$ into (A.5), we have

$$
\begin{aligned}
{[\dot{I}](t)=} & \lambda[S I](t)-\int_{0}^{t} n \gamma(a)\left\{\lambda[S I](t-a) e^{-\int_{0}^{a} n \gamma(s) d s}+\int_{0}^{a} \gamma(s) S i(t-a+s, s) e^{-\int_{s}^{a} n \gamma(w) d w} d s\right\} d a \\
& -\int_{t}^{\infty} n \gamma(a)\left\{\varphi(a-t) e^{-\int_{a-t}^{a} n \gamma(s) d s}+\int_{0}^{t} \gamma(a-t+s) S i(s, a-t+s) e^{-\int_{a-t+s}^{a} n \gamma(w) d w} d s\right\} d a \\
& +\int_{0}^{t} \gamma(a)\left\{\frac{[S](t-a) v(t) \sigma(a)}{\lambda[S S](t-a)[S I](t-a) v(t-a)}-\int_{0}^{a} \frac{\gamma(s) v(t) \sigma(a)}{i(t-a+s, s) v(t-a+s) \sigma(s)} d s\right\}^{-1} d a \\
& +\int_{t}^{\infty} \gamma(a)\left\{\frac{v(t) \sigma(a)}{\psi(a-t) \sigma(a-t)}-\int_{0}^{t} \frac{\gamma(a-t+s) v(t) \sigma(a)}{i(s, a-t+s) v(s) \sigma(a-t+s)} d s\right\}^{-1} d a .
\end{aligned}
$$


Similarly, using $[S I](t)=\int_{0}^{\infty} S i(t, a) d a$, from the fourth equation of system (2.2) we have

$$
\begin{aligned}
{[\dot{S I}](t)=} & \int_{0}^{\infty} \frac{\partial}{\partial t} \operatorname{Si}(t, a) d a \\
= & \int_{0}^{\infty}\left(-(\lambda+\mu+n \gamma(a)) \operatorname{Si}(t, a)-(\lambda+\mu) \operatorname{ISi}(t, a)+\gamma(a) \operatorname{Si}(t, a) S-\frac{\partial}{\partial a} \operatorname{Si}(t, a)\right) d a \\
= & -(\lambda+\mu)[S I](t)-\int_{0}^{\infty} n \gamma(a) \operatorname{Si}(t, a) d a-(\lambda+\mu) \frac{[S I]^{2}(t)}{[S](t)} \\
& +\int_{0}^{\infty} \gamma(a) \frac{S^{2}(t, a)}{i(t, a)} d a-S i(t, \infty)+\operatorname{Si}(t, 0) .
\end{aligned}
$$

Solving the first-order linear partial differential equation of $\operatorname{Si}(t, a)$ along characteristic lines, where the $\operatorname{Si}(t, a)$ is derived in detail in Appendix A, we have

$$
\operatorname{Si}(t, a)= \begin{cases}\left\{\frac{[S](t-a) v(t) \sigma(a)}{\lambda[S S](t-a)[S I](t-a) v(t-a)}-\int_{0}^{a} \frac{\gamma(s) \sigma(a) v(t)}{i(t-a+s, s) \sigma(s) v(t-a+s)} d s\right\}^{-1}, & t>a, \\ \left\{\frac{v(t) \sigma(a)}{\psi(a-t) \sigma(a-t)}-\int_{0}^{t} \frac{\gamma(a-t+s) v(t) \sigma(a)}{i(s, a-t+s) v(s) \sigma(a-t+s)} d s\right\}^{-1}, & t \leqslant a .\end{cases}
$$

Applying the formula (A.8) for $[S I](t)=\int_{0}^{\infty} S i(t, a) d a$, we have

$$
\begin{aligned}
{[S I](t)=} & \int_{0}^{t}\left\{\frac{[S](t-a) v(t) \sigma(a)}{\lambda[S S](t-a)[S I](t-a) v(t-a)}-\int_{0}^{a} \frac{\gamma(s) \sigma(a) v(t)}{i(t-a+s, s) \sigma(s) v(t-a+s)} d s\right\}^{-1} d a \\
& +\int_{t}^{\infty}\left\{\frac{v(t) \sigma(a)}{\psi(a-t) \sigma(a-t)}-\int_{0}^{t} \frac{\gamma(a-t+s) v(t) \sigma(a)}{i(s, a-t+s) v(s) \sigma(a-t+s)} d s\right\}^{-1} d a .
\end{aligned}
$$

Finally, using that along the characteristic lines, $\operatorname{Si}(t, \infty)=\operatorname{Si}(0, \infty) \approx \frac{\mathfrak{n}}{N}[S]_{0} \varphi(\infty)=0$ from the assumption, substituting (A.8) and the boundary condition of $\operatorname{Si}(t, a)$ into (A.7), we have

$$
\begin{aligned}
{[\dot{S I}](t)=} & \lambda \frac{n}{N}[S](t)[S I](t)-(\lambda+\mu)[S I](t)-(\lambda+\mu) \frac{[S I]^{2}(t)}{[S](t)} \\
& +\int_{0}^{t} \frac{\gamma(a)}{\lambda[S I](t-a) e^{-\int_{0}^{a} n \gamma(s) d s}+\int_{0}^{a} \gamma(s) S i(t-a+s, s) e^{-\int_{s}^{a} n \gamma(w) d w} d s} \\
& \times\left\{\frac{[S](t-a) v(t) \sigma(a)}{\lambda[S S](t-a)[S I](t-a) v(t-a)}-\int_{0}^{a} \frac{\gamma(s) v(t) \sigma(a)}{i(t-a+s, s) v(t-a+s) \sigma(s)} d s\right\}^{-2} d a \\
& +\int_{t}^{\infty} \frac{\gamma(a)}{\varphi(a-t) e^{-\int_{a-t}^{a} n \gamma(s) d s}+\int_{0}^{t} \gamma(a-t+s) S i(s, a-t+s) e^{-\int_{a}^{a}-t+s} n \gamma(w) d w d s} \\
& +\left\{\frac{v(t) \sigma(a)}{\psi(a-t) \sigma(a-t)}-\int_{0}^{t} \frac{\gamma(a-t+s) v(t) \sigma(a)}{i(s, a-t+s) v(s) \sigma(a-t+s)} d s\right\}^{-2} d a \\
& -\int_{0}^{t} n \gamma(a)\left\{\frac{[S](t-a) v(t) \sigma(a)}{\lambda[S S](t-a)[S I](t-a) v(t-a)}-\int_{0}^{a} \frac{\gamma(s) v(t) \sigma(a)}{i(t-a+s, s) v(t-a+s) \sigma(s)} d s\right\}^{-1} d a \\
& -\int_{t}^{\infty} n \gamma(a)\left\{\frac{v(t) \sigma(a)}{\psi(a-t) \sigma(a-t)}-\int_{0}^{t} \frac{\gamma(a-t+s) v(t) \sigma(a)}{i(s, a-t+s) v(s) \sigma(a-t+s)} d s\right\}^{-1} d a .
\end{aligned}
$$

